\title{
Avaliação do efeito hipoglicemiante de Cissus sicyoides em estudos clínicos fase II
}

\author{
Hosana Bandeira Santos, ${ }^{*}, 1$ João Modesto-Filho, ${ }^{2}$ Margareth de Fátima Formiga Melo \\ Diniz, ${ }^{1}$ Tereza Helena Cavalcanti de Vasconcelos, ${ }^{3}$ Francisco Santiago de Brito Pereira, ${ }^{4}$ \\ Josué do Amaral Ramalho, ${ }^{1}$ Jadson Gomes Dantas, ${ }^{1}$ Esther Bandeira Santos ${ }^{5}$
}

\author{
${ }^{1}$ Laboratório de Tecnologia Farmacêutica, Universidade Federal da Paraíba, Campus Universitário, \\ 58051-970 João Pessoa-PB, Brasil, \\ ${ }^{2}$ Departamento de Medicina Interna, Universidade Federal da Paraíba, Campus Universitário, \\ 58051-970 João Pessoa-PB, Brasil, \\ ${ }^{3}$ Departamento de Nutrição, Universidade Federal da Paraíba, Campus Universitário, \\ 58051-970 João Pessoa-PB, Brasil, \\ ${ }^{4}$ Hospital Universitário Lauro Wanderley, Universidade Federal da Paraíba, Campus Universitário, \\ 58051-970 João Pessoa-PB, Brasil, \\ ${ }^{5}$ Departamento de Odontologia, Universidade Federal da Paraíba, Campus Universitário, \\ 58051-970 João Pessoa-PB, Brasil
}

\begin{abstract}
RESUMO: O estudo objetivou realizar ensaios clínicos de fase II com o infuso das folhas de Cissus sicyoides L (Vitaceae) para investigar a eficácia terapêutica desse vegetal em voluntárias intolerantes à glicose $(\mathrm{GIG} n=14)$ e diabéticas $(\mathrm{GD} n=12)$ entre 30 e 59 anos de idade no Hospital Universitário Lauro Wanderley/UFPB. O chá foi preparado com $1 \mathrm{~g}$ do pó das folhas secas, diluído em $150 \mathrm{~mL}$ de água quente por 10 minutos (uso popular), dose única, por um período de 7 dias (fase aguda). No GIG verificou-se a interação entre a glicemia e a insulina através do Teste Oral de Tolerância à Glicose, sem e com a ingestão do infuso. A glicemia e insulinemia foram determinadas nos tempos $0,1,2$ e $3 \mathrm{~h}$. Para o GD avaliou-se a variação espontânea da glicemia (em jejum e em repouso nos tempos 0, 1, 2 e 3 h) e o perfil glicêmico (antes e $2 \mathrm{~h}$ após as refeições) na ausência e na presença do chá. No GIG, o chá das folhas teve atividade hipoglicemiante significativa aos 120 minutos, porém, não houve aumento da insulinemia, além da fisiológica, sugerindo que esse efeito não ocorreu por liberação ou secreção da mesma. No GD o infuso não apresentou efeito hipoglicemiante significativo.
\end{abstract}

Unitermos: Cissus sicyoides, Vitaceae, estudos clínicos, atividade hipoglicemiante.

\begin{abstract}
Evaluation of the hypoglycemic effect of Cissus sicyoides in phase II clinical trials". This study aimed to perform Phase II clinical trials with the infusion of the leaves of Cissus sicyoides L to investigate its therapeutic effectiveness in glucose-intolerant (GIG $\mathrm{n}=$ 14 ) and in type 2 diabetic (GD $n=12$ ) volunteers, 30 to 59 years old, at Hospital Universitário Lauro Wanderley/UFPB. The tea was prepared with $1 \mathrm{~g}$ of dust falling leaves diluted in $150 \mathrm{~mL}$ of hot water for 10 minutes (popular usage), in single doses, for 7 days (acute phase). In the GIG, the interaction between the glicemy and the insulin was verified through two oral tests of glucose tolerance, with and without infusion's ingestion. The glicemy and the insulinemy were determined at $0,1,2$, and 3 hours. For GD, the spontaneous variation of glicemy (during fasting and resting periods, at $0,1,2$, and 3 hours), and the glicemic profile (before and 2 hours after meals) were evaluated in the absence and in the presence of the tea. For GIG, the tea of the leaves revealed considerable hypoglycemic activity at 120 minutes; however there was no insulinemy increase, beyond the physiological, suggesting that this effect did not happen because of insulinemy's liberation or secretion. For GD, the tea did not demonstrate considerable hypoglycemic activity.
\end{abstract}

Keywords: Cissus sicyoides, Vitaceae, clinical trials, hypoglycemic activity.

\section{INTRODUÇÃO}

O Diabetes melito é uma disfunção metabólica de múltipla etiologia caracterizado por hiperglicemia crônica resultante da deficiência na secreção de insulina pelas células beta do pâncreas, na ação da insulina ou ambos (Nucci, 2003).

Essa patologia representa um dos mais 
importantes problemas de saúde pública alcançando expressiva importância como causa de doença e morte, em quaisquer que sejam os países ou raças consideradas. A importância do diabetes tem crescido em virtude das suas repercussões sociais e econômicas, o que traduz-se tanto por mortes prematuras, absenteísmo e incapacidade para o trabalho, quanto por custos associados ao seu controle ou ao tratamento de suas complicações, principalmente as de natureza ocular, renal e vascular (Brasil, 1993; Barceló et al., 2003).

Segundo a Organização Mundial de Saúde, o número de diabéticos no mundo é de 180 milhões. O Brasil possui 5,7 milhões de diabéticos e está em $7^{\circ}$ lugar no ranking mundial (Sociedade Brasileira de Diabetes, 2003; Federação Internacional de Diabetes, 2003).

$\mathrm{Na}$ Paraíba, de acordo com o Ministério da Saúde, existem 135 mil diabéticos e a grande maioria, 37,5 mil, está concentrada na cidade de João Pessoa. O Estado e a capital, João Pessoa, apresentam aumento na tendência da mortalidade por diabetes na população de 40 anos e mais, semelhante ao ocorrido no Brasil e na região Nordeste (Brasil, 2006).

Com a crescente demanda por medicamentos e considerando-se o seu elevado custo, a busca de fontes alternativas e a implementação de projeto sistemático para o estudo e validação do uso de plantas medicinais parecem mais que justificáveis (Barbosa-Filho et al., 1991; Almeida et al., 2001; Rocha et al., 2005; Albuquerque \& Hanazaki, 2006; Silva et al., 2006; Biavatti et al., 2007).

O gênero Cissus é o maior da família Vitaceae, com cerca de 350 espécies distribuídas entre as Américas, a Ásia e a Austrália. Estudos realizados com algumas espécies de Cissus têm revelado várias atividades farmacológicas, dentre as quais destacam-se as atividades: antioxidante e antimicrobiana (Murthy et al., 2003; Silva et al., 2007), inibidor da enzima acetilcolinesterase (Barbosa-Filho et al., 2006), hipoglicemiante (Barbosa et al., 2002), na prevenção da osteoporose (Shirwaikar et al., 2003), entre outras. O objeto do presente estudo é a Cissus sicyoides L, uma trepadeira conhecida popularmente como anil trepador, uva brava, cipó-pucá ou insulina vegetal (Agra et al, 2007). As folhas são empregadas externamente contra reumatismo, abscessos e a infusão de folhas e do caule são utilizadas na inflamação muscular, na hipotensão e como ativadora da circulação sangüínea. A espécie em referência vem sendo muito empregada pela população para o tratamento do diabetes, sendo por isso conhecida como "insulina vegetal" dando motivo para estudos botânicos, químicos e farmacológicos no Brasil e no exterior (Silva et al., 1996; Mori et al, 2001; Beltrame et al., 2001; Pepato et al., 2003, Barbosa-Filho et al., 2005).

O estudo de fase II é realizado com um pequeno número de pacientes portadores da patologia que se pretende estudar e com administração do produto.
O que se busca nessa fase é: estabelecer a eficácia clínica e a incidência de reações adversas; definir os esquemas posológicos mais apropriados; e coletar dados farmacológicos, farmacocinéticos e metabólicos para aprimorar a utilização do fármaco. Ao fim dessa fase, os pesquisadores terão reunido cerca de $65 \%$ de todo conhecimento sobre o fármaco, depois de dois anos de pesquisa ao custo de $15 \%$ do total dos recursos empregados (Oliveira, 2006).

Diante da inexistência de ensaios clínicos com a Cissus sicyoides publicados até o momento, propôse a realização de estudos farmacológicos clínicos de fase II com o infuso das folhas da referida planta com a finalidade de investigar a eficácia terapêutica desse vegetal em humanos intolerantes à glicose e em diabéticos do tipo 2 .

\section{METODOLOGIA}

\section{Material botânico}

As folhas de Cissus sicyoides L. foram obtidas no horto de plantas medicinais do Laboratório de Tecnologia Farmacêutica "Delby Fernandes de Medeiros" da Universidade Federal da Paraíba (UFPB), onde a planta é cultivada. Amostra representativa encontra-se no herbário Lauro Pires Xavier, localizado no Centro de Ciências Exatas e da Natureza (CCEN/ UFPB).

\section{Obtenção dos "sachês do chá" de Cissus sicyoides}

As folhas foram desidratadas em estufa com ar circulante, a $40{ }^{\circ} \mathrm{C}$, por $72 \mathrm{~h}$, e trituradas em moinho tipo Harley, obtendo-se um rendimento médio de $12 \%$. As folhas secas foram encaminhadas para a APLAFComércio e Indústria Ltda, localizada no município de Itatiba - São Paulo, para a confecção dos "sachês do chá" da planta, que continham 1 grama do pó das folhas.

\section{Controle sanitário das folhas de Cissus sicyoides}

As análises microbiológicas foram realizadas no Laboratório de Tecnologia de Alimentos do Centro de Tecnologia/UFPB. A legislação referente ao controle microbiológico em alimentos é a RDC no 12 de 02 de janeiro de 2001 da ANVISA (Brasil, 2001), porém, essa legislação só preconiza ensaios para coliformes fecais. Para um maior controle de qualidade do material botânico a ser utilizado pelos pacientes foram realizadas as contagens de bactérias mesófilas facultativas viáveis, de coliformes totais e fecais e de bolores e leveduras. $\mathrm{O}$ tratamento das amostras e os métodos utilizados para a realização das análises foram realizados de acordo com o Instituto Adolfo Lutz, 1985.

\section{Estudos clínicos}


O estudo foi desenvolvido no período de janeiro de 2005 a setembro de 2006 envolvendo pacientes, de ambos os sexos, intolerantes à glicose e pacientes diabéticos tipo 2, entre 30 e 59 anos de idade.

Os ensaios clínicos foram realizados no ambulatório do Hospital Universitário Lauro Wanderley (HULW) da UFPB, com a infra-estrutura necessária ao desenvolvimento da pesquisa, inclusive para atender eventuais problemas resultantes da mesma. Os eletrocardiogramas foram realizados no Setor de Cardiologia do Ambulatório do referido Hospital e os exames laboratoriais e hematológicos no Laboratório de Análises Clínicas do HULW/UFPB.

O estudo teve a participação livre e espontânea de acordo com as diretrizes e normas regulamentadoras de pesquisas envolvendo seres humanos, contidas nas Resoluções oㅡ 196/96 (Brasil, 1996) e 251/97 (Brasil, 1997) do Conselho Nacional de Saúde (CNS) do Ministério da Saúde e após ter sido submetido e devidamente autorizado pelo Comitê de Ética do HULW/UFPB. Durante todo o curso dos experimentos, os voluntários foram instruídos a comunicarem, aos pesquisadores, qualquer sinal ou sintoma clínico que porventura vierem a apresentar.

Foram considerados critérios de exclusão, os voluntários que eram analfabetos (por não terem condições de preencher o formulário necessário na fase aguda), que demonstraram alterações laboratoriais clinicamente importantes nos exames de análises clínicas, que revelavam disfunção hepática, renal, diabetes tipo 1 ou diabetes tipo 2 com uma glicemia de jejum maior de $180 \mathrm{mg} / \mathrm{dL}$, alterações patológicas graves, grávidas, nutrizes, alcoólatras ou que estivessem em uso de medicação para diabetes. Também foram excluídos aqueles pacientes que eram domiciliados geograficamente distantes do centro de pesquisa (HULW) não garantindo assim a estabilidade no acompanhamento clínico.

A seleção dos pacientes ocorreu através de amostragem não aleatória, por conveniência, entre homens e mulheres, na faixa etária entre 30 e 59 anos que procuraram o Ambulatório de Endocrinologia e de nutrição do HULW como também os que freqüentavam os Centros de Referência em Diabetes tanto do Estado como do Município de João Pessoa. Da mesma forma, foram recrutados pacientes através de campanhas realizadas dentro da UFPB entre os funcionários da mesma e em Programas de Ação Social nas comunidades do Porto do Capim e Cidade Verde em Mangabeira VIII. Dos pacientes contactados inicialmente $(30$ homens e 65 mulheres), apenas 26 mulheres concluíram o tratamento.

As voluntárias pré-selecionadas foram distribuídas em 2 grupos: Grupo das Intolerantes à Glicose (GIG, $n$ =14) e o Grupo das Diabéticas (GD, $\mathrm{n}=12)$.

As diagnosticadas como intolerantes à glicose foram avaliadas através do Teste Oral de Tolerância à Glicose (TOTG) que é uma prova provocativa na qual se administra uma dose relativamente grande de glicose para estimular os mecanismos homeostáticos do organismo (Ravel, 1997). As voluntárias compareceram ao Hospital às $7 \mathrm{~h}$ da manhã após jejum de $12 \mathrm{~h}$ para a coleta sangüínea basal (tempo 0), em seguida, administrou-se, por via oral, $75 \mathrm{~g}$ de açúcar (dextrosol) em $300 \mathrm{~mL}$ de água e 1, 2, e $3 \mathrm{~h}$, depois se determinou a glicemia e a insulinemia. Um segundo TOTG foi agendado e 30 minutos antes da carga de glicose a voluntária ingeriu o chá de Cissus sicyoides preparado com $1 \mathrm{~g}$ do pó das folhas secas diluído em $150 \mathrm{~mL}$ de água quente (uso popular), em caneca padronizada. Para verificação da resistência insulínica realizou-se o método HOMA (Homeostasis Assessment Model) O HOMA é um modelo matemático que permite estimar a presença de resistência à insulina (HOMAR = insulinemia $\mathrm{x}$ glicemia/ 22,5) como também de avaliar a função das células beta do pâncreas (HOMAB $=20 \mathrm{x}$ insulinemia/ glicemia -3,5) (Wallace et al., 2004).

Nas diabéticas verificou-se a variação espontânea da glicemia na ausência e na presença do chá. A avaliação foi realizada em domicílio, estando as pacientes em jejum e em repouso, através do glicosímetro Optium da Abbott Laboratories. Com o auxílio de um lancetador, o sangue era coletado nos tempos $0,1,2$ e 3 horas, por 3 dias seguidos e os valores da glicemia eram anotados em formulário próprio. Após esse período as pacientes foram instruídas a verificar a glicemia do tempo 0 (ao despertar), em seguida, ingerir o infuso e proceder da mesma forma.

Para a realização do perfil glicêmico, as diabéticas, fizeram punção digital antes das refeições e duas horas após as mesmas, anotando as glicemias obtidas. A avaliação ocorreu por três dias seguidos sem a ingestão do chá e três dias com a ingestão do mesmo. Após esse procedimento, as participantes foram instruídas a tomarem o infuso por sete dias sempre no período da manhã e em jejum, quando compareciam ao Hospital para as diversas avaliações.

\section{RESULTADOS E DISCUSSÃO}

Os resultados das análises microbiológicas realizadas nos "sachês" (Tabela 1) mostram que os mesmos estavam dentro da Legislação vigente e apropriados ao consumo humano.

Na RDC no 12 de 02/01/2001 não contem todos os parâmetros microbiológicos para alimentos como os chás e isso deve ser atribuído ao fato de que segundo a ANVISA na Resolução no 23/2000, que foi revogada pela RDC no 278 de 22/09/2005 (Brasil, 2005), os chás são alimentos dispensados da obrigatoriedade de registro.

Conforme os resultados das duas curvas glicêmicas mostrados na Tabela 2, observou-se que quando as pacientes ingeriram o chá este foi capaz 
de reduzir os valores de glicemia em todos os tempos ocorrendo diferença significativa apenas no tempo de duas horas e não houve aumento da insulina, além do esperado fisiologicamente, donde se conclui que o mecanismo de ação da planta na redução da glicemia, através da prova funcional aplicada, não envolveu aumento na liberação desse hormônio.

Algumas plantas produzem efeitos periféricos (extra-pancreáticos) sobre o metabolismo dos carboidratos sem alterar os níveis de insulina (Bragança, 1996; Hernandez-Galicia et al., 2002).

Pepato e Baviera (2003) e Viana et al. (2004) ao realizarem estudos pré-clínicos com extrato aquoso da Cissus sicyoides sugeriram que o mecanismo de ação que causou efeito hipoglicemiante nos animais avaliados não se assemelha ao das sulfoniluréias ou à insulina e que a planta deve agir de forma semelhante às biguanidas.

Bragança (1996) e Pereira (1997) relatam os possíveis mecanismos de ação hipoglicemiante das plantas, que incluem: 1) diminuição da digestão e absorção de carboidratos; 2) estímulo à síntese e/ ou secreção de insulina; 3) aumento da afinidade e/ou concentração de receptores de insulina; 4) interferência sobre a biotransformação da insulina; 5) ações periféricas sobre o metabolismo dos carboidratos, como a inibição da gliconeogênese, estímulo à glicogênese, etc; 6) efeito protetor sobre a célula beta da ilhota de Langerhans; 7) inibição da célula alfa da ilhota de Langerhans ou de agentes hiperglicemiantes; 8) efeito benéfico ou protetor sobre a nefro, a angio, a retino e/ ou neuropatias diabéticas; 9) substituição do açúcar da dieta; 10) fornecimento de nutrientes.

Em estudos pré-clínicos, Mori et al., (2001) verificaram atividade hipoglicemiante com o extrato da folhas de Cissus sicyoides. Verificaram que a glicemia plasmática dos animais diminuiu significativamente 1 hora após uma carga de sacarose e concluíram que a ingestão de "insulina" impede o aumento da glicose depois da refeição.

Barbosa et al. (2002), administraram extrato aquoso ( 5 folhas $=12 \mathrm{~g}$ em $1000 \mathrm{~mL}$ de água por 1 minuto) de Cissus verticillata, em substituição da água, em ratos normoglicêmicos por 30 dias. Constataram efeito hipoglicemiante que foi atribuído à presença de flavonóides encontrados em grande quantidade no chá e de canferol livre possivelmente acompanhado de seu glicosídeo, o que justificaria, ao menos em parte, a atividade hipoglicemiante do vegetal in vivo.

Já Vasconcelos (2004) e Vasconcelos et al. (2007), observaram em estudos pré-clínicos utilizando a fração aquosa das folhas de Cissus sicyoides em camundongos Swiss machos normais que a glicemia teve tendência à queda sem caracterizar diferença significativa entre o tempo basal e sete dias de tratamento e em humanos, clinicamente normais, de ambos os sexos, o infuso não foi capaz de diminuir a glicemia, significativamente, num período de oito semanas.

Os resultados do jejum do HOMA R das pacientes avaliadas (Tabela 2) estão dentro dos valores até agora relatados na literatura para pacientes normais, ou seja, mesmo ao serem diagnosticadas como intolerantes à glicose, não apresentaram resistência à insulina, por esse método de avaliação.

Tambascia et al., (2000) relataram que alguns pacientes com graus muito altos de resistência insulínica, como é observado na obesidade, não mostram nenhum nível de intolerância à glicose devido a uma habilidade muito eficiente de secretar insulina levando a um estado de hiperinsulinemia compensatória.

O índice HOMA - Homeostasis Assesment Model, descrito por Matthews et al., (1985), é um modelo matemático que tem sido validado diante do padrão ouro, o clamp euglicêmico hiperinsulinêmico, e vem sendo utilizado por vários autores dando sustentação ao seu uso principalmente em estudos epidemiológicos (Bonora et al., 2000; Haffner et al., 1996; Ikeda et al., 2001; Straczkowski et al., 2003; Yokoyama, et al., 2004; Straczkowski et al., 2004). O HOMA pode ser usado com os pacientes em jejum como em estudos fisiológicos onde esses parâmetros são avaliados mediante prova funcional como o TOTG (Wallace et al., 2004).

Oliveira et al., 2005 publicaram extensa revisão sobre o HOMA na prática clínica e concluíram que realmente existe a falta de um valor de corte estabelecido como referência para classificar os resultados dos pacientes. Em diversos estudos os valores variavam de 1,0 a 3,0 para o HOMA R: para pacientes hígidos HOMA R = 1,0 (Matthews et al., 1985); estudos com 90 pacientes japoneses hígidos e 281 diabéticos tipo 2 o índice encontrado foi 3,0 (Kuwana, 2002); para 6511 americanos o índice encontrado foi de 2,8 $\pm 2,4$ (Bravata et al., 2004) ; para chilenos não obesos o HOMA R foi igual a 1,96 $\pm 0,57$ (Acosta et al., 2002); em turcos o índice foi de 2,24 $\pm 1,26$ (Gokcel et al., 2003) e para Taniguchi et al., (2000) o índice foi de 2,5 em estudo que avaliou a existência de duas variantes de não-obesos levemente diabéticos com e sem a resistência à insulina. Quanto ao HOMA B ficou estipulado por Matthews et al., (1985), que em pacientes hígidos, com menos de 35 anos de idade e peso corporal normal o valor seria igual a $100 \%$, ou seja, os pacientes tinham $100 \%$ de funcionamento das células beta.

Ao serem avaliadas quanto à resistência insulínica (RI), pacientes com a síndrome dos ovários

Tabela 1. Número mais provável de coliformes totais e fecais. Contagens de bactérias mesófilas facultativas viáveis e de bolores e leveduras realizadas nos saches de Cissus sicyoides.

\begin{tabular}{ccc|c}
\hline Análises & Resultados & Legislação & \\
\hline Coliformes a $35^{\circ} \mathrm{C}(\mathrm{NMP} / \mathrm{g})$ & $2,4 \times 10$ & - & \\
Coliformes a $45^{\circ} \mathrm{C}(\mathrm{NMP} / \mathrm{g})$ & 4 & Até 10 & \\
Bactérias mesófilas (UFC/g) & $3,0 \times 10^{5}$ & - & \\
Bolores e leveduras (UFC/g) & $3,0 \times 10^{3}$ & $5,0 \times 10^{3}$ & \\
\hline & & \\
\hline & & Rev. Bras. Farmacogn. & 73 \\
& Braz J. Pharmacogn. & \\
& 18(1): Jan./Mar. 2008 &
\end{tabular}


Tabela 2. Valores das variáveis: glicemia $(\mathrm{mg} / \mathrm{dL})$, insulina $(\mathrm{mmol} / \mathrm{L})$, homaR e homaB em teste oral de tolerância à glicose segundo a condição.

\begin{tabular}{llcc}
\hline \multirow{2}{*}{ Variável } & \multicolumn{1}{c}{$\begin{array}{c}\text { Tempo de } \\
\text { Avaliação }\end{array}$} & $\begin{array}{c}\text { Sem chá }(\mathrm{n}=14) \\
\text { Média } \pm \mathrm{DP}^{(1)}\end{array}$ & $\begin{array}{c}\text { Com chá }(\mathrm{n}=14) \\
\text { Média } \pm \text { DP }\end{array}$ \\
\cline { 2 - 4 } Glicemia & Jejum & $106,21 \pm 10,19$ & $106,36 \pm 9,48$ \\
& Após 1 hora & $196,79 \pm 25,58$ & $194,07 \pm 39,91$ \\
& Após 2 horas & $165,07 \pm 19,75$ & $139,71 \pm 36,00^{*}$ \\
& Após 3 horas & $107,57 \pm 22,57$ & $99,29 \pm 18,18$ \\
Insulina & & & $7,23 \pm 3,85$ \\
$($ Basal $<20)$ & Jejum & $7,88 \pm 5,38$ & $53,50 \pm 30,93$ \\
$($ Após $<150)$ & Após 1 hora & $52,01 \pm 30,38$ & $79,76 \pm 71,93$ \\
& Após 2 horas & $85,39 \pm 62,63$ & $28,76 \pm 25,14$ \\
Após 3 horas & $30,27 \pm 30,95$ & $1,92 \pm 1,06$ \\
& & & $27,15 \pm 19,89$ \\
& Jejum & $2,07 \pm 1,44$ & $31,40 \pm 33,94$ \\
& Após 1 hora & $25,91 \pm 15,83$ & $7,46 \pm 6,64$ \\
& Após 2 horas & $35,44 \pm 26,26$ & \\
Após 3 horas & $8,77 \pm 10,05$ & $60,92 \pm 35,17$ \\
& & & $146,86 \pm 68,02$ \\
& Jejum & $68,69 \pm 50,90$ & $364,19 \pm 273,47$ \\
& Após 1 hora & $138,60 \pm 79,36$ & $301,08 \pm 261,98$ \\
\hline
\end{tabular}

(*) Diferença significante a 5,0\% entre os grupos.

(1) DP - Desvio padrão.

policísticos (SOP) com peso normal (G1) e sobrepeso/ obesas (G2), o HOMA R foi 1,9 e 3,6, respectivamente. O HOMA B do G1 foi 144,2 e o do G2 foi de 265 (Kuba et al., 2006).

A variação espontânea da glicemia apresentou redução da glicemia quando as pacientes ingeriram o chá; apesar de a maior diferença ter ocorrido 1 hora após a ingestão do mesmo, essa diferença não foi significativa em nenhum dos tempos (Tabela 3).

$\mathrm{Na}$ avaliação do perfil glicêmico (Tabela 4) observou-se que as pacientes tiveram a glicemia reduzida após a ingestão do chá, porém, sem diferença significativa entre as condições; a maior redução ocorreu no tempo de duas horas após o café da manhã.

A literatura relata que tem sido investigado possível efeito hipoglicemiante com as folhas Cissus sicyoides havendo relatos contraditórios quanto a essa atividade. Silva et al. (1996), Beltrame et al. (2001), Beltrame et al. (2002), Vasconcelos, (2004) e Lima (2007) não observaram essa atividade diferentemente de Mori et al. (2001), Barbosa et al. (2002), Pepato et al. (2003) e Viana et al. (2004).

Sabe-se que são vários os fatores que interferem num estudo clínico como a dieta, a amostragem, o estresse diário, a variabilidade individual dos pacientes, a dificuldade em se utilizar placebo com chás (forma mais utilizada pelos pacientes), pois os chás têm, em geral, sabor próprio afetando assim, estudo cego e também a interferência de medicamentos.
Tabela 3. Valores da glicemia (mg/dL) segundo a condição no jejum e após uma, duas e três horas em repouso.

\begin{tabular}{lcc}
\hline & \multicolumn{2}{c}{ Condição } \\
Tempo de & Sem chá $(\mathrm{n}=12)$ & Com chá $(\mathrm{n}=12)$ \\
Avaliação & Média $\pm \mathrm{DP}^{(1)}$ & Média $\pm \mathrm{DP}$ \\
\hline Jejum & $125,19 \pm 26,91$ & $117,81 \pm 26,09$ \\
Após 1 hora & $124,33 \pm 29,96$ & $114,86 \pm 22,43$ \\
Após 2 horas & $121,22 \pm 35,01$ & $116,44 \pm 39,16$ \\
Após 3 horas & $117,83 \pm 37,87$ & $112,28 \pm 40,01$ \\
\hline
\end{tabular}

(1) - DP - Desvio padrão.

Tabela 4. Valores da glicemia (mg/dL) segundo a condição para o jejum, duas horas após o café, antes do almoço, duas horas após o almoço, antes do jantar e duas horas após o jantar.

\begin{tabular}{lcc}
\hline \multirow{2}{*}{$\begin{array}{c}\text { Tempo de } \\
\text { Avaliação }\end{array}$} & \multicolumn{2}{c}{ Condição } \\
\cline { 2 - 3 } & Sem chá $(\mathrm{n}=12)$ & Com chá $(\mathrm{n}=12)$ \\
\cline { 2 - 3 } & & \\
\hline Jejum & $117,69 \pm 25,06$ & $119,25 \pm 29,13$ \\
2 h após café & $148,47 \pm 55,44$ & $138,44 \pm 44,91$ \\
Antes do almoço & $116,11 \pm 37,26$ & $115,22 \pm 38,56$ \\
2 h após almoço & $132,47 \pm 40,12$ & $128,17 \pm 31,90$ \\
Antes do jantar & $125,06 \pm 30,97$ & $124,00 \pm 23,15$ \\
2 h após jantar & $145,64 \pm 40,45$ & $146,69 \pm 35,93$ \\
\hline
\end{tabular}

(1) DP - Desvio padrão.

\section{CONCLUSÃO}

No estudo clínico de fase aguda, através das curvas glicêmicas traçadas, o chá das folhas de Cissus 
sicyoides teve efeito hipoglicemiante significativo aos 120 minutos, porém, não houve aumento da insulinemia, além da fisiológica, sugerindo que esse efeito não ocorreu por liberação ou secreção da mesma. O chá das folhas quando utilizado no teste de variação espontânea da glicemia e no perfil glicêmico nas pacientes diabéticas, não apresentou efeito hipoglicemiante significativo como também não foi observada essa atividade em 7 dias de administração do chá. No presente estudo, não foi comprovada a afirmação popular que cita a Cissus sicyoides como "insulina vegetal". Sugere-se a continuidade dos estudos clínicos mediante a avaliação da fase crônica.

\section{AGRADECIMENTOS}

Os autores agradecem à Fundação de Apoio à Pesquisa do Estado da Paraíba (FAPESQ) por ter viabilizado recursos para a realização desse estudo.

\section{REFERÊNCIAS}

Acosta BAM, Escalona OM, Maiz GA, Pollak CF, Leighton PF 2002 Determinación del índice de resistência insulínica mediante HOMA em uma población de la Region Metropolitana de Chile. Rev Med Chile 130:1227-1231.

Agra MF, França PF, Barbosa-Filho JM 2007. Synopsis of the plants known as medicinal and poisonous in Northeast of Brasil. Rev Bras Farmacogn 17: 114-140.

Albuquerque UP, Hanazaki N 2006. As pesquisas etnodirigidas na descoberta de novos fármacos de interesse médico e farmacêutico: fragilidades e pespectivas. Rev Bras Farmacogn 16(Supl.): 678-689.

Almeida RN, Navarro DS, Barbosa-Filho JM 2001. Plants with central analgesic activity. Phytomedicine 8: 310-322.

Barbosa WLR, Santos WRA, Pinto LN, Tavares ICC 2002. Flavonóides de cissus verticillata e a atividade hipoglicemiante do chá de suas folhas. Rev Bras Farmacogn 12: 13-15.

Barbosa-Filho JM, Agra MF, Oliveira RAG, Paulo MQ, Troling G, Cunha EVL, Ataide JR, Bhattacharyya J 1991. Chemical and pharmacological investigation of Solanum species of Brazil - a search for solasodine and other potentially useful therapeutic agents. Mem I Oswaldo Cruz 86: 189-191.

Barbosa-Filho JM, Vasconcelos THC, Alencar AA, Batista LM, Oliveira RAG, Guedes DN, Falcão HS, Moura MD, Diniz MFFM, Modesto-Filho J 2005. Plants and their active constituints from South, Central and North América with hypoglicemic activity. Rev Bras Farmacogn 15: 392-413.

Barbosa-Filho JM, Medeiros KCP, Diniz MFFM, Batista LM, Athayde-Filho PF, Silva MS, Cunha EVL, Almeida JRGS, Quintans-Júnior LJ 2006. Natural products inhibitors of the enzyme acetylcholinesterase. Rev Bras Farmacogn 16: 258-285.

Barceló A, Aedo C, Rajpathak S 2003. The cost of diabetes in Latin America and the Caribbean. Bull World Health Org 81: 19-27.

Beltrame FL, Sartoretto JL, Bazotte RB, Cuman RN, Cortez
DAG 2001. Estudo fitoquímico e avaliação do potencial antidiabético do Cissus sicyoides L. (Vitaceae). Quim Nov 24: 783-785.

Beltrame FL, Ferreira AG, Cortez DAG 2002. Coumarin glycoside from Cissus sicyoides. Nat Prod Lett 16: 213-216.

Biavatti MW, Marensi V, Leite SN, Reis A 2007. Ethnopharmacognostic survey on botanical compendia for potential cosmeceutic species from Atlantic Forest. Rev Bras Farmacogn 17: 640-653.

Bonora L, Targher G, Alberiche M, Bonadonna RC, Saggiani F, Zenere MB, Monauni T, Muggeo M 2000. Homeostasis model assessment closely mirrors the glucose clamp technique in the assessment of insulin sensitivity: studies in subjects with various degrees of glucose tolerance and insulin sensitivity. Diabetes Care 23: 57-63.

Bragança LAR 1996 Plantas medicinais antidiabéticas: uma abordagem multidisciplinar. Niterói - Rio de Janeiro: UFF. 300p.

Brasil 1993. Ministério da Saúde. Secretaria de Assistência à Saúde. Departamento de Assistência à Saúde. Coordenação de Doenças Crônico - Degenerativas. Brasília - DF: Manual de Diabetes. 92 p.

Brasil 1996. Ministério da Saúde. Conselho Nacional de Saúde. Resolução n¹96/1996. Aprova diretrizes e normas regulamentadoras de pesquisa envolvendo seres humanos. Brasília - DF, 10 de outubro de 1996.

Brasil 1997. Ministério da Saúde. Conselho Nacional de Saúde. Resolução $n^{\circ} 251 / 1997$. Aprova normas de pesquisa envolvendo seres humanos para a área temática de pesquisa com novos fármacos, medicamentos, vacinas e testes diagnósticos. Brasília - DF, 07 de agosto de 1997.

Brasil 2001. Ministério da Saúde. Agência Nacional de Vigilância Sanitária (ANVISA). Resolução RDC $\mathrm{n}^{\circ} 12 / 2001$. Dispõe sobre o regulamento técnico sobre padrões microbiológicos para alimentos. Brasília - DF, 02 de janeiro de 2001.

Brasil 2005. Ministério da Saúde. Agência Nacional de Vigilância Sanitária (ANVISA). Resolução RDC N ${ }^{\circ}$ 278/2005. Dispõe sobre o Regulamento Técnico sobre o Manual de Procedimentos Básicos para Registro e Dispensa da Obrigatoriedade de Registro de Produtos Pertinentes à Área de Alimentos. Brasília - DF, 22 de setembro de 2005.

Brasil 2006. Ministério da Saúde. Sistema Nacional de Vigilância em Saúde: relato de situação: Paraíba. Brasília - DF.

Bravata DM, Wells C, Concato J, Kernan WN, Brass LM, Gulanski BI 2004. Two measures of insulin sensitivity provided similar information in a U. S. population. $J$ Clin Epidemiol 57: 1214-1217.

Federação Internacional de Diabetes 2003. El Diabetes Atlas. $56 \mathrm{p}$.

Gokcel A, Batali M, Tarim E, Bagis T, Gumurdulu Y, Karakose H, Yalcin F, Akbaba M, Guvener N 2003. Diabetes Obes Metab 5: 126-130.

Haffner SM, Kennedy E, Gonzalez C, Stern MP, Miettinen H 1996. A prospective analysis of the HOMA model: the Mexico City Diabetes Study. Diabetes Care 19: 1138-1141.

Hernandez-Galicia E, Aguilar-Contreras A, AguilarSantamaria L, Roman-Ramos R, Chavez-Miranda 
AA, Garcia-Vega LM, Flores-Saenz JL, AlarconAguilar FJ 2002. Studies on hypoglicemic activity of Mexican medicinal plants. Proc West Pharmacol Soc 45: 118-124.

Ikeda Y, Suehiro T, Nakamura T, Kumon Y, Hashimoto K 2001. Clinical significance of the insulin resistance index as assessed by homeostasis model assessment. Endocr J 48: 81-86.

Instituto Adolfo Lutz 1985. Normas Analíticas do Instituto Adolfo Lutz: métodos químicos e físicos para análises de alimentos. São Paulo. 371 p.

Kuba VM, Cavalieri PM, Christóforo AC, Faria Junior R, Caetano R, Coeli CM, Athayde A 2006. Resistência insulínica e perfil metabólico em pacientes com síndrome dos ovários policísticos de peso normal e sobrepeso/obesiodade. Arq Bras Endocr Metab 50: 1026-1033.

Kuwana B 2002. Reference value and cut-off value for diagnosis of insulin resistance in type 2 diabetes mellitus. Off J Jpn Soc Lab 50: 398-403.

Lima CMBL 2007. Avaliação da toxicidade aguda e crônica do extrato hidroalcoólico das folhas de Cissus sicyoides L. João Pessoa. 108p. Dissertação de Mestrado Universidade Federal da Paraíba.

Matthews DR, Hosker JP, Rudenski AS, Naylor BA, Treacher DF, Turner RC 1985. Homeostasis model assessment: insulin resistance and beta-cell function from fasting plasma glucose and insulin concentrations in man. Diabetol 28: 412-419.

Mori T, Nishikawa Y, Takata Y, Kashiuchi N, Ishihara N 2001. Effect of insulina leaf extract on development of diabetes: comparison between normal, streptozotocininduced diabetic rats and hereditary diabetic mice. $J$ Jpn Soc Nut Food Sci 54: 197-203.

Murthy KNC, Vanitha A, Swamy MM, Ravishankar GA 2003. Antioxidant and antimicrobial activity of Cissus quadrangularis L. J Med Food 6: 99-105.

Nucci LB 2003. A Campanha Nacional de Deteç̧ão de Diabetes Mellitus: Cobertura e Resultados Glicêmicos. Porto Alegre. 120p. Tese de Doutorado - Faculdade de Medicina da Universidade Federal do Rio Grande do Sul.

Oliveira EP, Souza MLA, Lima MDA 2005. Índice HOMA (homeostasis model assessment) na prática clínica: uma revisão. J Bras Patol Med Lab 41: 237-243.

Oliveira GG 2006. Ensaios Clínicos: Princípios e Prática. Brasília: Anvisa. 328p.

Pepato MT, Baviera AM, Vendramini RC, Perez MPMS, Kettelhutt IC, Brunetti IL 2003 Cissus sicyoides (princess vine) in the long-term treatment of streptozotocin-diabetic rats. Biot Appl Bioch 37: $15-20$

Pereira NA 1997. Plants as hypoglycemic agents. Cienc Cult 49: 354-358

Radikova Z 2003. Assessment of insulin sensitivity/resistance in epidemiological studies. Endocr Reg 37: 189-194.

Ravel R 1997. Laboratório Clinico: Aplicações Clínicas dos Dados Laboratoriais. Rio de Janeiro: Ed. Guanabara Koogan S.A. 616p.

Rocha LG, Almeida JRGS, Macedo RO, Barbosa-Filho JM 2005. A review of natural products with antileishmanial activity. Phytomedicine 12: 514-535.
Silva GA, Akisue G, Oga S 1996. Ensaio farmacológico de ação hipoglicemiante dos extratos fluidos de Cissus sicyoides L. Rev Bras Farmacogn 5: 144 -155.

Silva MIG, Gondim APS, Nunes IFS, Sousa FCF 2006. Utilização de fitoterápicos nas unidades básicas de atenção à saúde da família no município de Maracanaú (CE). Rev Bras Farmacogn 16: 455-462.

Silva L, Oniki GH, Agripino DG, Moreno PRH, Young MCM, Mayworm MAS, Ladeira AM 2007. Biciclogermacreno, resveratrol e atividade antifúngica em extratos de folhas de Cissus verticillata (L.) Nicolson \& Jarvis (Vitaceae). Rev Bras Farmacogn 17: 361-367.

Sociedade Brasileira de Diabetes 2002. Consenso Brasileiro Sobre o Diabetes: diagnóstico e classificação do diabetes melito e tratamento do diabetes melito tipo 2. Rio de Janeiro: 75 p.

Straczkowski M, Stepien A, Kowalska I, Topolska J, Kinalska I 2003. An assessment of insulin during exercise training program in obese women. Comparison of simple indices with hyperinsulinemic euglycemic clamp technique. Pol Arch Med Wew 109: 483-488.

Straczkowski M, Stepien A, Kowalska I, Kinalska I 2004. Comparison of simple indices of insulin sensitivity using the euglycemic hyperinsulinemic clamp technique. Med Sci Mon 10: 80-84.

Tambascia MA, Geloneze B, Rodovalho SO, Pareja JC, Franco DM, Repetto EM 2000. Beta cell disfunction as the main factor to determine glucose intolerance in insulin resistance patients. International Diabetes Federation Congress. México.

Taniguchi A, Fukushima M, Sakai M, Kataoka K, Nagata I, Doi K, Arakawa H, Nagasaka S, Tokuyama K, Nakai Y 2000. The role of the body mass index and triglyceride levels in identifying insulin-sensitive and insulin-resistant variants in Japanese noninsulin-dependent diabetic patients. Metabolism 49: 1001-1005.

Vasconcelos THC 2004. Ensaios toxicológicos pré-clínico e clínico com as folhas da Cissus sicyoides L. João Pessoa 178p.Tese de doutorado - Universidade Federal da Paraíba.

Vasconcelos THC, Modesto-Filho J, Diniz MFFM, Santos HB, Aguiar FB, Moreira PVL 2007. Estudo toxicológico pré-clínico agudo com o extrato hidroalcoólico das folhas de Cissus sicyoides L. (Vitaceae). Rev Bras Farmacogn 17: 583-591.

Viana GSB, Medeiros ACC, Lacerda AMR, Leal KAM, Vale TG, Matos FJA 2004. Hipoglycemic and anti-lipemic effects of the aqueous extract from Cissus sicyoides. BMC Pharm 4: 1-7.

Wallace TM, Levy JC, Mattheews DR 2004. Use and abuse of HOMA modeling. Diabetes Care 27: 1487-1495.

Yokoyama H, Emoto M, Fujiwara S, Motoyama K, Morioka T, Komatsu M, Tahara H, Koyama H, Shoji T, Inaba M, Nishizawa Y 2004. Quantitative insulin sensitivity check index and the reciprocal index of homeostasis model assessment are useful indexes of insulin resistance in type 2 diabetic patients with wide range of fasting plasma glucose. J Clin Endocr Metab 89: 1481-1484. 